\title{
WINGATE ANAEROBIC TEST: ADDITIONAL PHYSIOOLOGICAL INTERPRETATION
}

\author{
Dorothea Stefanova, Borislava Petrova
}

National Sports Academy "Vassil Levski”, Sofia, Bulgaria

\begin{abstract}
The Wingate Anaerobic Test (WAnT) is the most commonly used test for the assessment of anaerobic power and anaerobic capacity of athletes. The well known parameters recorded during the WAnT routine procedure in our opinion could be subjected to additional analysis generating substantial additional physiological data. The aim of this study was to develop a new, easy to set up method estimating the relationship between the contribution of the anaerobic and aerobic energy systems in athletes performing WAnT. A parallel registration of WAnT parameters and the oxygen consumption breath by breath was done. Thus, the indicators for the activity of the aerobic and anaerobic systems, ensuring the physical effort in each segment (in seconds) of the test, were calculated. The proposed method was introduced and

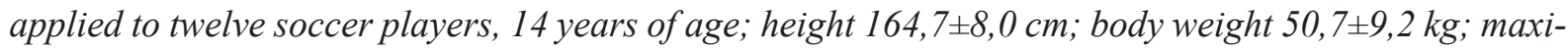

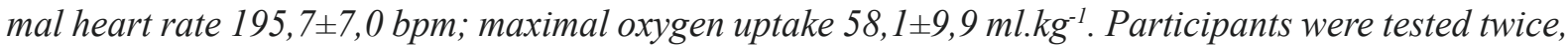
before and after 5 months specialized training program focussed on the improvement of speed/power and endurance. Statistically significant progress was found in: Total power output by 16,4\%; $\mathrm{O}_{2}$ deficit by 32,4\%; $\mathrm{O}_{2}$ deficit $/ \mathrm{O}_{2}$ excess by 80,3\% in favor of the deficit. It was established that the differences in the calculated physiological parameters during the performance of the WAnT before and after the fivemonth training program are more pronounced compared to the ergometric ones, especially with regard to the participation of each system in energy processes. The results indicate that the proposed physiological parameters are of high sensitivity and reflect strong associations with the metabolic changes in response to the training modalities. The method is easy to implement, allowing quick analysis of the individual results and its application in exercise physiology laboratories and the sports practice could be beneficial.
\end{abstract}

Key words: Wingate Anaerobic Test, oxygen uptake, oxygen deficit, power output

\section{INTRODUCTION}

The Wingate Anaerobic Test (WAnT) is the most commonly used test for the direct assessment of anaerobic power and an indirect assessment of the anaerobic capacity of athletes. Anaerobic capacity is an important parameter that determines fitness and the performance of the athletes not only in short-term high-intensity sprint/power sports (100 m, 200 $\mathrm{m}$ ) but also in sports requiring multiple sprints ( soccer, basketball, volleyball), as well as in sports disciplines of longer duration. Deter- mining the amount of anaerobic energy supply during physical efforts is difficult from a methodological point of view. The most commonly used method for determination of the anaerobic capacity is the MaximalAccumulated Oxygen Deficit Method (MAOD) (Medbo,1988; Medbo, Tabata,1989,1993). Green (1995) defined MAOD as an estimation of the total amount of ATP resynthesised via anaerobic metabolism by the whole body during short duration high-intensity exercise. In the original protocol used by Medbo 
et al, (1988) a linear relationship between power output (PO) and oxygen consumption $\mathrm{VO}_{2}$ (Medbo, Tabata,1989;1993) is established for each individual. This relationship is extrapolated to supramaximal exercise intensities and the $\mathrm{VO}_{2}$ demand corresponding to supramaximal workloads is predicted. When this $\mathrm{VO}_{2}$ demand is multiplied by the duration of the exercise bout, the accumulated $\mathrm{VO}_{2}$ demand is estimated. The MAOD is calculated by subtracting the accumulated $\mathrm{VO}_{2}$, measured during the exercise bout, from the estimated accumulated $\mathrm{VO}_{2}$ demand (Noordhof, 2010). The main barrier to the wide use of maximal accumulated oxygen deficit is the considerable time required to complete several sub- and supra-maximal exercise sessions (Zagatto, 2016). The method required the participant to perform a $\mathrm{VO}_{2}$ maximal test, several (the best 10) submaximal pre-tests of 4 minutes duration and a supramaximal exercise test.

The researchers' efforts were focussed on finding ways to reduce these time-consuming procedures (Zagatto, 2016; Bertuzzi et al, 2010).

Few studies pointed out that deficiency of the method is that MAOD power are capacity values which do not provide information about the distribution of anaerobic energy during exercise (Noordhof et al, 2013; Seresse et al, (1988).

Beneke R et al, (2002) explored for the first time the energy requirements of WAnT based on all energy contributors such as high energy phosphates, glycolysis, and oxidative metabolism. For this purpose, they measured $\mathrm{VO}_{2}$ data during the test and 30 minutes after its termination, blood lactate concentration at short intervals during the recovery period and the ergometric parameters from WAnT. The results supported the finding that WAnT metabolism is highly anaerobic, showing that $80 \%$ of the energy turnover during WAnT is derived from anaerobic alactic and lactic acid metabolism (predominantly by glycolysis).
When using computerized Wingate test procedure, the key parameters are automatically recorded: Peak Power in Wats; Relative Peak Power per body weight; Average power, defined as Anaerobic Capacity; Anaerobic Fatigue index.

These parameters are well known and used for the analysis of the Wingate test performance. However, in our opinion the data obtained could be subjected to additional analysis resulting in the generation of new substantial physiological data set. On the basis of these new physiological information the assessment of the athletes ' potential in successfully competing in a particular sport and the athletes' response to the training protocols would be more precise.

The aim of this study was to develop a new, easy to set up method estimating the relationship between the contribution of the anaerobic and aerobic energy systems in athletes performing Wingate anaerobic test.

We hypothesized that using this method, the potentially beneficial changes in the relationship between anaerobic and the aerobic capacity in response to sport training could be identified and assessed objectively.

\section{METHODOLOGY}

\section{Description of the method}

In order to develop a new testing method for analysis of the Wingate test results we had to apply a specific methodological approach which consists of a parallel registration of WAnT parameters and the oxygen consumption breath by breath of the participants during the performance of the test.

We have to emphasize that the new method requires the registration of the data for the power output (W. $\mathrm{s}^{-1}$ ) and oxygen consumption $\left(\mathrm{VO}_{2} \mathrm{ml} \cdot \mathrm{s}^{-1}\right)$ for each second during the period of thirty seconds when performing the WAnT. The registered results and the subsequent calculations of one participant as an example are presented in Table 1 as an example. 
Table 1. Ergometic and respiratory indices co-registered and calculated when performing Wingate test

\begin{tabular}{|c|c|c|c|c|c|c|c|c|c|c|c|c|c|c|}
\hline $\mathrm{s}$ & W & $\begin{array}{c}\mathrm{VO} 2 \\
\text { uptake } \\
\left(\mathrm{ml.s}^{-1}\right)\end{array}$ & $\begin{array}{l}\text { VO2 de- } \\
\text { mand } \\
\left(\mathrm{ml} . \mathrm{s}^{-1}\right)\end{array}$ & $\begin{array}{c}\mathrm{O}_{2} \\
\text { deficit } \\
\left(\mathrm{ml} . \mathrm{s}^{-1}\right)\end{array}$ & s & W & $\begin{array}{c}\mathrm{VO} 2 \\
\text { uptake } \\
\left(\mathrm{ml} . \mathrm{s}^{-1}\right)\end{array}$ & $\begin{array}{l}\text { VO2 de- } \\
\text { mand } \\
\left(\mathrm{ml} . \mathrm{s}^{-1}\right)\end{array}$ & $\begin{array}{c}\mathrm{O} 2 \\
\text { deficit/ } \\
\text { excess } \\
\left(\mathrm{ml} . \mathrm{s}^{-1}\right)\end{array}$ & $\mathbf{s}$ & W & $\begin{array}{c}\text { VO2 } \\
\text { uptake } \\
(\mathrm{ml.s})\end{array}$ & $\begin{array}{l}\text {-VO2 de } \\
\text { mand } \\
\left.(\mathrm{ml.s})^{-1}\right)\end{array}$ & $\begin{array}{l}\text { excess } \\
\left.\mathrm{m}\}_{4} s^{-}\right)\end{array}$ \\
\hline 1 & 594,8 & 17,7 & 37,1 & $-19,4$ & 11 & 540,9 & 21,4 & 34,6 & $-13,2$ & 21 & 441,7 & 38,2 & 29,8 & 8,4 \\
\hline 2 & 629,3 & 19,2 & 38,8 & $-19,6$ & 12 & 555,7 & 18,8 & 35,3 & $-16,5$ & 22 & 429,3 & 36,0 & 29,2 & 6,8 \\
\hline 3 & 631,6 & 19,2 & 38,9 & $-19,7$ & 13 & 529,3 & 29,9 & 34,0 & $-4,1$ & 23 & 426,3 & 36,0 & 29,1 & 7,0 \\
\hline 4 & 645,4 & 21,5 & 39,6 & $-18,0$ & 14 & 522,8 & 30,7 & 33,7 & $-3,0$ & 24 & 396,0 & 37,7 & 27,6 & 10,1 \\
\hline 5 & 607,2 & 18,3 & 37,7 & $-19,5$ & 15 & 508,6 & 30,7 & 33,0 & $-2,4$ & 25 & 398,1 & 44,1 & 27,7 & 16,4 \\
\hline 6 & 628,5 & 18,3 & 38,7 & $-20,5$ & 16 & 518,0 & 28,8 & 33,5 & $-4,6$ & 26 & 384,0 & 38,6 & 27,1 & 11,5 \\
\hline 7 & 609,9 & 21,2 & 37,9 & $-16,6$ & 17 & 478,4 & 33,3 & 31,6 & 1,8 & 27 & 375,2 & 35,5 & 26,6 & 8,9 \\
\hline 8 & 592,5 & 18,9 & 37,0 & $-18,1$ & 18 & 484,7 & 34,1 & 31,9 & 2,2 & 28 & 379,8 & 35,5 & 26,9 & 8,6 \\
\hline 9 & 560,6 & 18,9 & 35,5 & $-16,6$ & 19 & 458,1 & 34,1 & 30,6 & 3,5 & 29 & 371,4 & 37,6 & 26,5 & 11,1 \\
\hline 10 & 583,9 & 21,4 & 36,6 & $-15,2$ & 20 & 433,7 & 35,9 & 29,4 & 6,5 & 30 & 364,4 & 42,4 & 26,1 & 16,3 \\
\hline Sum & & & & & & & & & & & 15080,1 & 873,9 & 981,8 & \\
\hline
\end{tabular}

$W$ and $\mathrm{VO}_{2}$ uptake are registered by computers

$\mathrm{VO}_{2}$ demand $=\left(\left(W^{*} 0,239\right) / 5\right)+\mathrm{VO}_{2}$ ml. $\mathrm{s}^{-1}$ from last $5 \mathrm{~s}$ of acceleration phase (here 8,7$)$;

$\mathrm{VO}_{2}$ deficit or $\mathrm{VO}_{2}$ excess $=\mathrm{VO}_{2}$ uptake- $\mathrm{VO}_{2}$ demand

From the obtained data, the oxygen demand for the performance at the given load has to be calculated. This requires the determination of the oxygen equivalent of the respective watts values, converted into calories (e.g. $1 \mathrm{~W}=0,239$ cal. $\left.\mathrm{s}^{-1}\right)$.

It is known that during a supramaximal exercise, besides limited muscle creatine phosphate reserves, only carbohydrates (glucose, mainly derived from muscle glycogen reserves) deliver energy, so that $1 \mathrm{ml} \mathrm{O}_{2} \cdot \mathrm{s}^{-}$ 1 consumed during WAnT releases energy equal to 5,047 cal.s $\mathrm{s}^{-1}$ or energy of $1 \mathrm{cal} . \mathrm{s}^{-1}$ is produced approximately by $0,2 \mathrm{ml} \mathrm{O}_{2} \cdot \mathrm{s}^{-1}$. In addition to the obtained values, the mean values of $\mathrm{VO}_{2} \mathrm{ml} . \mathrm{s}^{-1}$ from the 5 seconds accelera- tion phase of each individual (e.g. $=8,7 \mathrm{ml} \cdot \mathrm{s}^{-1}$ ) has been included in the calculations shown in Table 1. Obviously, the outcome from the subtraction the $\mathrm{VO}_{2}$ demand from the actual measured $\mathrm{VO}_{2}$ consumption represents the $\mathrm{O}_{2}$ deficit (see the results presented with a minus sign in Table 1). From the example presented in Table 1, we observed that the first 12 seconds of the test are highly anaerobic. The results indicated that the anaerobic component lasts up to 16 seconds, but from 12 th to 16 th seconds it is much less pronounced. It should be pointed out that in the second half of the test oxidative metabolism component has shown noticeable increase (Figure 1).

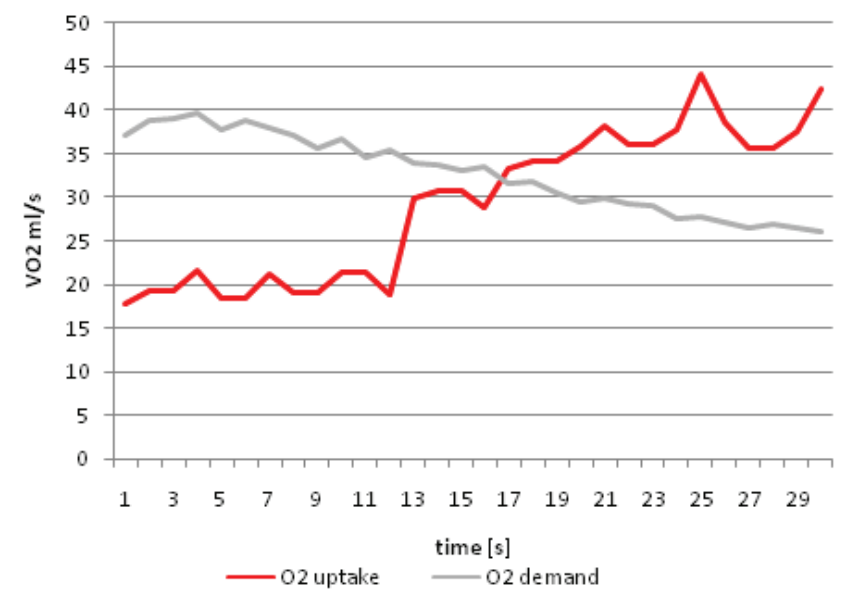

Fig. 1. Oxygen uptake (ml.s-1) and Oxygen demand (ml.s-1) during WAnT 
Using the above-mentioned data, additional information can be obtained about the relationship between aerobic and anaerobic energy supply of physical effort in WAnT by calculating the parameters presented in Table 2. Since the test aims to determine the anaerobic ability of the person, it is of particular importance to determine the relationship between oxygen deficit (as a marker for anaerobic power) and the excess amount oxy- gen needed during the test performance to pay off the deficit. It is expected that as a result of speed and power training, the $\mathrm{O}_{2}$ deficit will increase and its payout during the test will begin later. The data of the Watts values obtained when performing the anaerobic component and the percentage contribution to the Total power output (W30 s) can also be added to the Wingate ergometric data.

Table 2. Physiological parameters calculated on the basis of the results presented in Table 1.

\begin{tabular}{lc}
\hline Calculated parameters & value \\
\hline (Total power output $(\mathrm{W} 30 \mathrm{~s}$ & 15080,1 \\
\hline (Total O, uptake $(\mathrm{ml} / 30 \mathrm{~s}$ & 873,9 \\
\hline (O2 deficit $(\mathrm{ml} / 30 \mathrm{~s}$ & 226,9 \\
\hline ( O2 excess $(\mathrm{ml} / 30 \mathrm{~s}$ & 119,0 \\
\hline (\%) O2 deficit/O2 excess & 190,7 \\
\hline Watts performed anaerobic & 4746,9 \\
\hline (\%) W anerobic/TotalW & 31,5 \\
\hline
\end{tabular}

\section{Participants}

Twelve young soccer players (14 years of age; height $164,7 \pm 8,0 \mathrm{~cm}$; body weight $50,7 \pm 9,2 \mathrm{~kg}$; maximal heart rate $195,7 \pm 7,0 \mathrm{bpm}$; maximal oxygen uptake $2893,9 \pm 654,9 \mathrm{ml} \cdot \mathrm{min}^{-1} ; 58,1 \pm 9,9 \mathrm{ml}$. $\mathrm{kg}^{-1}$ ), who were familiar with exhaustive exercises, volunteered to participate in this study. The subjects were fully informed about the details and discomfort associated with the experiments before the participants and their parents gave their informed consent to volunteer.

\section{Exercise testing procedures}

The participants were tested twice at the beginning (Testing I) and after 5 months of training period (Testing II). Testing I consisted of two separate sessions with an interval of 48 hours between sessions. In the first session the participants performed a maximal incremental exercise test to measure the maximal oxygen uptake ( $\mathrm{VO}_{2} \max$ test). In the second session the 30second Wingate Anaerobic test (WAnT) was performed. Testing II included only the WAnT performance.

\section{Maximal incremental exercise test}

The maximal incremental exercise test for the determination of $\mathrm{VO}_{2} \max$ was carried out on an treadmill H/P/Cosmos, (Nussdorf-Transtein, Germany) under a modified ramping protocol "Balke for children ", with constant speed $8.4 \mathrm{~km} / \mathrm{h}$, step duration of 30 seconds, initial slope of $0 \%$ and an increase in the slope of each step by $0,6 \%$ up to exhaustion. In order to attain maximal values participants received verbal encouragement to continue for as long as possible. Oxygen uptake $\left(\mathrm{VO}_{2}\right)$ was measured breath-by-breath throughout the test using a portable gas analyzer MetaMax 3B-R2 (Leipzig, Germany) and was subsequently averaged over $30 \mathrm{~s}$ intervals. The $\mathrm{VO}_{2} \max$ was recorded as the oxygen uptake measured during the last $30 \mathrm{~s}$ of the test.

\section{0-Wingate Anaerobic Test}

The 30-WAnT was performed on a mechanically braked cycle ergometer (Monark, 894 E, Stockholm, Sweden) as described by Inbar et al. (Inbar O,1996). 
In addition, the subjects were connected to the portable gas analyzer MetaMax 3B-R2 (Germany) to allow the respiratory parameters to be recorded simultaneously with the power output realized during the test.

The test started with a standardized warming up of 5 min cycling at $60 \mathrm{~W}$ and 60 revolution/min including two sprints, each lasting $3 \mathrm{~s}$, performed at the end of the $3 \mathrm{rd}$ and the 5 th min. The seat height and handle were individually adjusted for the subject's comfort, with the legs being nearly fully extended during each pedal revolution. After 5-min rest the subjects were instructed to pedal as fast as possible for $30 \mathrm{~s}$. A resistance corresponding to $7.5 \%$ of the body mass was applied after an acceleration phase lasting $5 \mathrm{~s}$. Verbal feedback to the time remaining was provided at 15,10 , and $5 \mathrm{~s}$ of remaining time while verbal encouragement was given. The subjects continued pedaling after completion of the test with no load for several minutes to cool down.

\section{Statistics}

Results are expressed as mean $\pm \mathrm{SD}$. In view of the relatively small sample size, the statistical significance of the study was calculated with the $\mathrm{X}^{2}$-criterion of Pearson (Chi Test - Excel). Statistical significance was accepted at the $\mathrm{P}<0.001$ level. In spite of its qualities, the Chi Test does not indicate which of the indicators used has the advantage over others, nor which athlete has shown the best results as an outcome of the conducted training program. For this purpose, we used the percentage differences in each individual between the testings, and the average values for the entire team were calculated with the formula:

$\sum\left(\mathrm{T}_{\mathrm{II}} / \mathrm{T}_{\mathrm{I}}-1\right)_{*} 100 / \mathrm{n}$, or average $\sum\left(\mathrm{T}_{\mathrm{II}} / \mathrm{T}_{\mathrm{I}}-\right.$ 1) $* 100 / \mathrm{n}$

where $\mathrm{T}_{\mathrm{II}}$ (Testing II) and $\mathrm{T}_{\mathrm{I}}$ (Testing I) are the data for every parameter of an individual, divided by the sample size (n).

\section{Research design}

The young soccer player performed the WAnT twice - at the beginning of the preparatory period of training (Test I) and at the beginning of the competitive period (Test II).

Experimental training protocols for improvement the speed/power and endurance of the recruited soccer players were developed and applied. Additional intensive workloads were performed in the main part of some training activities, when marked fatigue was accumulated. This approach was considered to be appropriate for developing speed endurance in groups of young athletes (Peev, 2017).

It is expected that as a result of speed and power training, the $\mathrm{O}_{2}$ deficit will increase and its payout during the test will begin later. The data of the Watts values obtained when performing the anaerobic component and the percentage contribution to the Total power output (W30 s) can also be added to the Wingate ergometric data.

The proposed method was introduced and applied to soccer players subjected to testing procedures at the high-performance exercise physiology laboratory at the Center for Scientific and Applied Rsearch in Sport at National Sports Academy, Sofia, in order to assess the impact of the 5 months training program on young soccer players.

\section{RESULTS}

The mean values of the parameters reported during the routine performance of the 30 second Wingate anaerobic Test are shown in Table 3 and those obtained by examining the physiological parameters characterizing the type of energy supply for this type of physical effort are presented in Table 4. 
Table 3. PeakPower output, Average power and Fatigue index (PD\%) in response to Wingate Test before (Test I) and after (Test II) five months of speed/ power and endurance training in young soccer players (mean values $\pm S D$ ).

\begin{tabular}{cccccc}
\hline Parameter & Test & Mean & SD & \% Dif II-I & Chi Test \\
\hline \multirow{2}{*}{ (PP (W } & I & 742,2 & 152,3 & \multirow{2}{*}{ (I,5 } & $\alpha<0,001$ \\
& II & 817,9 & 148,8 & & \\
\hline \multirow{2}{*}{ (AP (W } & I & 540,7 & 103,4 & \multirow{2}{*}{15,1} & $\alpha<0,001$ \\
& II & 620,4 & 115,6 & & \\
\hline & I & 50,0 & 10,0 & & \\
\% PD & II & 50,3 & 10,2 & 1,3 & $\alpha<0,548$
\end{tabular}

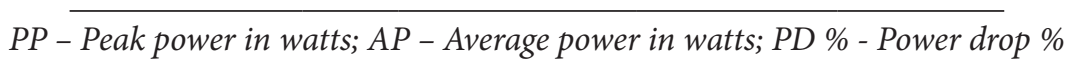

The 14 years old soccer players are dis- that the percentage differences found in this tinguished by their high accomplishment in study and used to assess the effect of the trainperforming of the WAnT. Peak Power (PP), ing are in fact in a very narrow range (between Anaerobic Power (AP) and Power Drop \% 11,3\% and 15,1\%). Therefore, it is not possi$(\mathrm{PD} \%)$ were significantly close to adult scores. ble to give priority to any one of the parameThe data in Table 3 showed a statistically sig- ters in order to evaluate the results objectively. nificant improvement in power output after the five months training program compared to those obtained before the beginning of the training, with the only exception the fatigue index $(\alpha<0,548)$. However, it is noteworthy

Figure 2 illustrates the activity of the respiratory system during the 30 -second supraximal load, where $\mathrm{O}_{2}$ uptake is still present from it's beginning and continues to grow to the end.

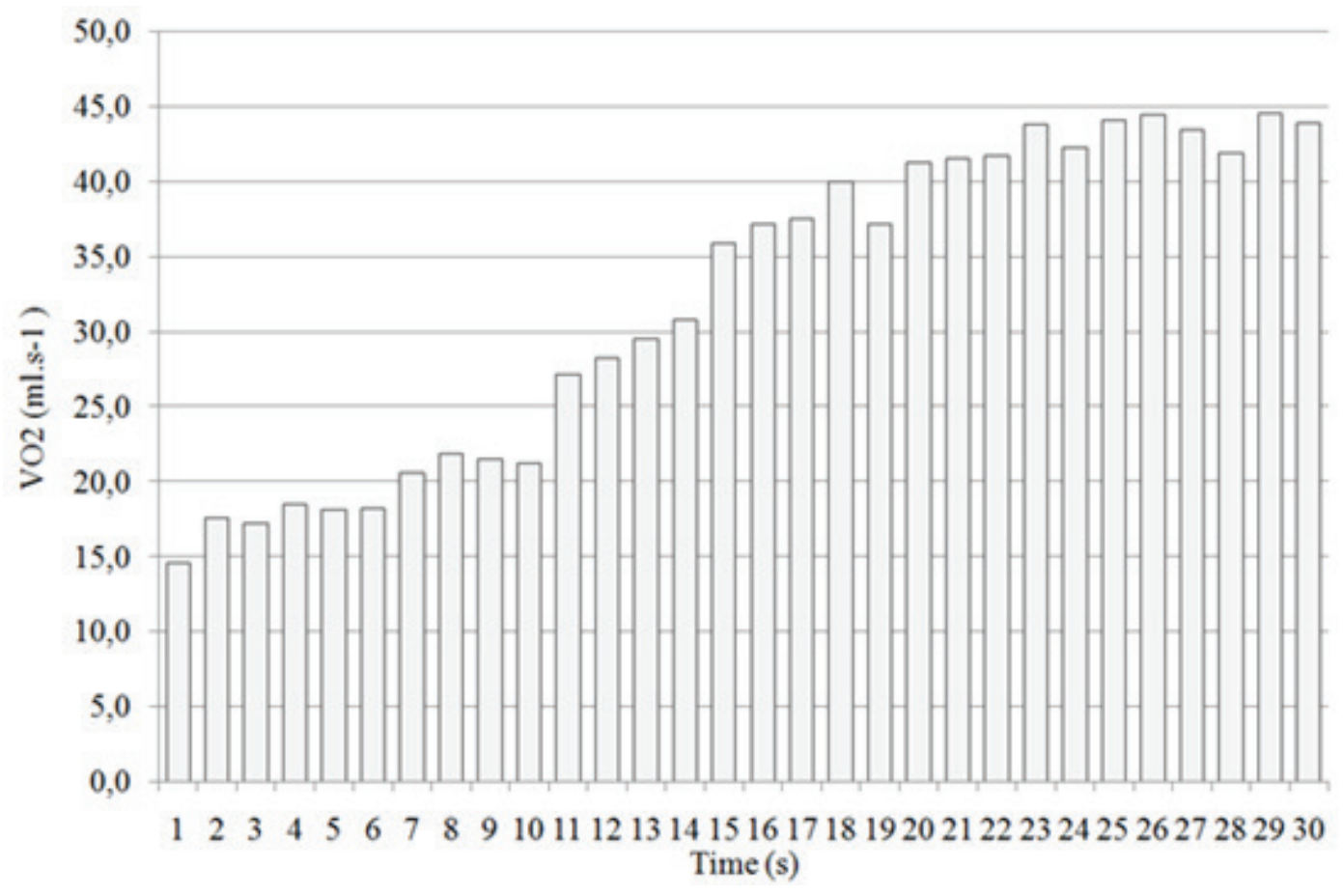

Fig. 2. $\mathrm{O}_{2}$ uptake (ml.s $\mathrm{s}^{-1}$ ) of young soccer players during Wingate Anaerobic Test. 
A high Chi Test difference was observed increased by $16.4 \%$ compared to Test I, but (Table 4) between the two investigations in all of the introduced physiological parameters, reflecting the impact of the fulfilled specific training program. Furthermore, the differences vary within a very wide range - from $6,9 \%$ the increase in $\mathrm{O}_{2}$ uptake was more than twice lower $-6.9 \%$. The $\mathrm{O}_{2}$ deficit is $32.4 \%$ higher after the performance of the 5-month training which reflects in the very small $\mathrm{O}_{2}$ excess $(9.1$ $\%)$ at Test II. to $80,3 \%$. In Test II total power output was

Table 4. Physiological parameters for assessment of the anaerobic and aerobic energy contribution during Wingate test (mean values \pm SD) and the difference between Test I and Test II (II-I \%) in response to five weeks of speed/power and endurance training.

\begin{tabular}{|c|c|c|c|c|c|}
\hline Calculated parameters & Test & $\operatorname{Mean}(\mathrm{n} 12)$ & SD & $\%$ II-I & Chi Test \\
\hline \multirow[t]{2}{*}{ Total power output (W 30s) } & I & 15667,6 & 2911,7 & & \\
\hline & II & 18194,8 & 3348,8 & 16,4 & $a<0,001$ \\
\hline \multirow[t]{2}{*}{ Total $\mathrm{O}_{2}$ uptake (30 s) } & I & 959,0 & 193,9 & & \\
\hline & II & 1004,4 & 149,5 & 6,9 & $a<0,001$ \\
\hline \multirow[t]{2}{*}{ Oxygen deficit (ml) } & I & 280,1 & 78,2 & & \\
\hline & II & 370,8 & 124,2 & 32,4 & $a<0,001$ \\
\hline \multirow[t]{2}{*}{ Oxygen excess (ml) } & I & 126,5 & 49,8 & & \\
\hline & II & 118,1 & 72,3 & 9,1 & $a<0,001$ \\
\hline \multirow[t]{2}{*}{$\mathrm{O}_{2}$ deficit $/ \mathrm{O}_{2}$ excess $(\%)$} & I & 251,4 & 105,9 & & \\
\hline & II & 419,7 & 243,8 & 80,3 & $a<0,001$ \\
\hline \multirow[t]{2}{*}{$\mathrm{W}$ performed anaerobic } & I & 5860,4 & 1635,0 & & \\
\hline & II & 7757,7 & 2599,3 & 32,4 & $a<0,001$ \\
\hline \multicolumn{6}{|l|}{$\mathrm{W}$ anaerobic/Total W (\%) } \\
\hline & $\mathrm{I}$ & 37,1 & 6,1 & 14,6 & $a<0,001$ \\
\hline
\end{tabular}

The relationships between anaerobic and aerobic participation in the test are illustrated in Figures 3 and 4. At the start of Test II, energy needs are satisfied with the provision of significantly more anaerobic energy, which continues until the $19^{\text {th }}$ second. In comparison, when performing Test I where the $\mathrm{O}_{2}$ deficiency is lower anaerobic work is terminated at the $15^{\text {th }}$ second (Figure 3 ). The observed $80,3 \%$ increase between Test I and II in $\mathrm{O}_{2}$ deficit $/ \mathrm{O}_{2}$ excess $(\%)$ is in favor of the deficit. The impact of the training is also reflected in the $32.4 \%$ increase in anaerobic power output during Test II. The ratio of the anaerobically produced watts to the total power output is increased by $14.5 \%$. 


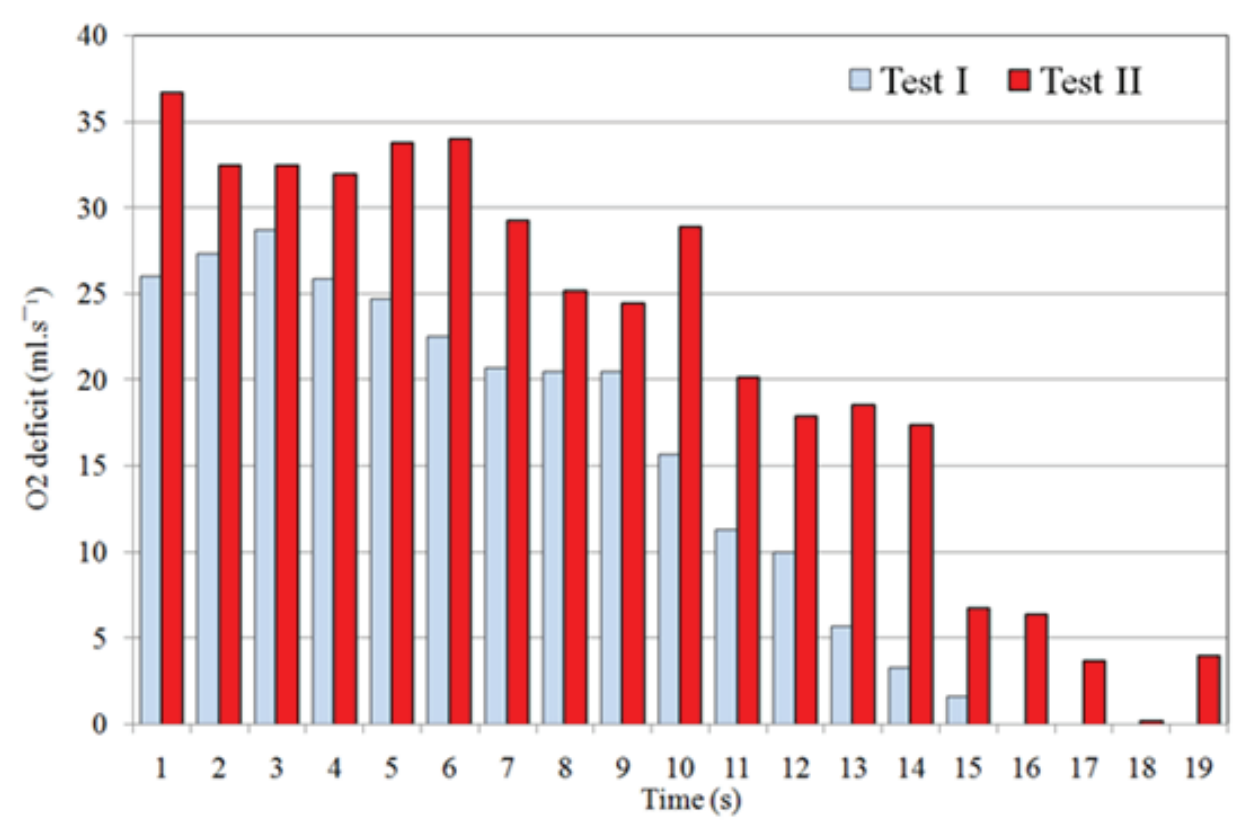

Fig. 3. Differences between the O2 deficit before (Test I) and after (Test II) five months training program in young soccer players.

Note: Test II was performed with a larger and longer accumulation of $\mathrm{O} 2$ deficit, in comparison with Test I.

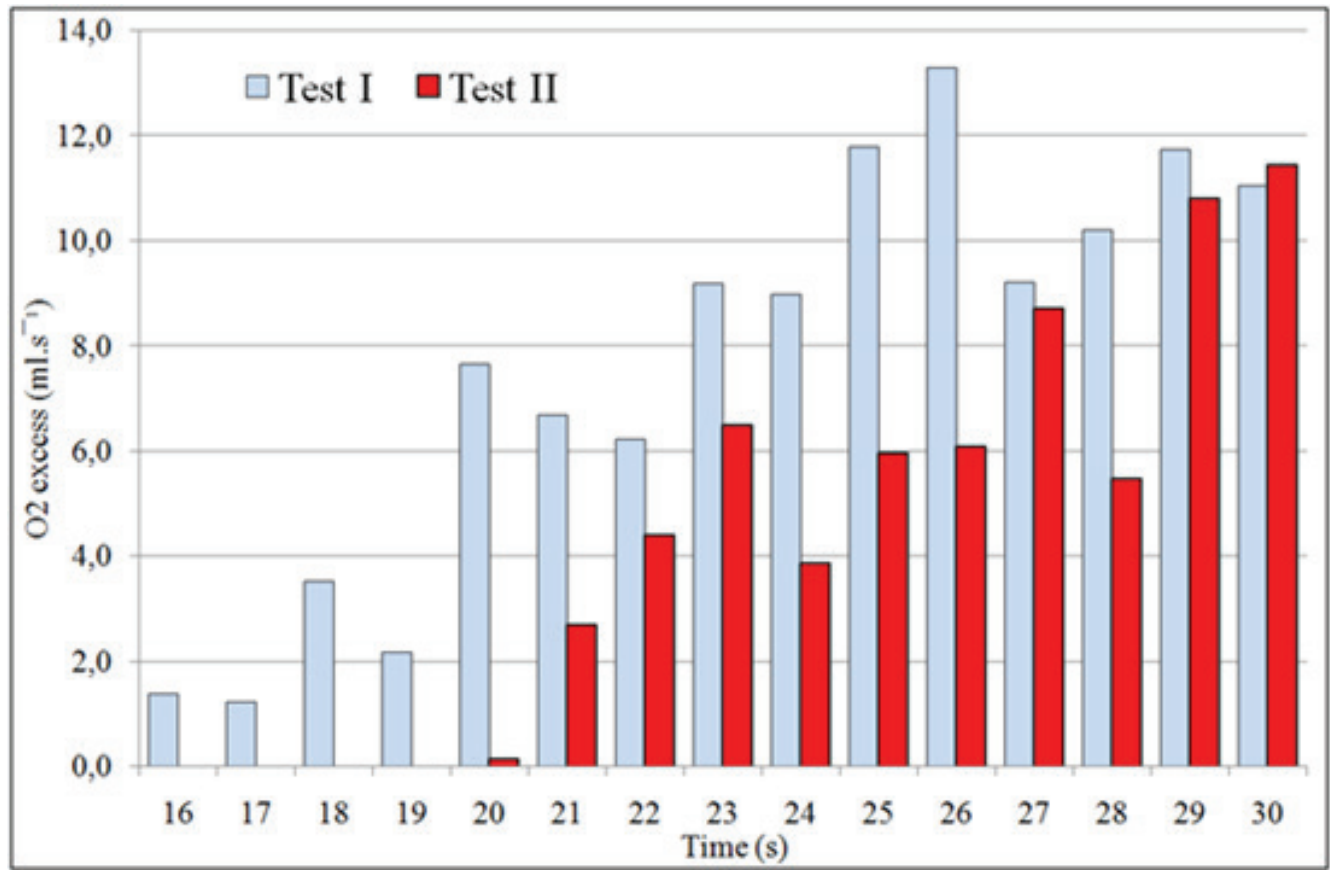

Fig. 4. Payment of the $\mathrm{O}_{2}$ deficit through the excess of $\mathrm{O}_{2}$ before (Test I) and after (Test II) five month training period of young soccer players

Note: The payment of $\mathrm{O}_{2}$-deficit during Test II begins later and $\mathrm{O}_{2}$-excess is with a smaller volume. 


\section{DISCUSSION}

This study investigated the relations between anaerobic and aerobic performance of pubertal (age 14) male soccer player and the method that we offered for calculation of the physiological parameters has shown considerable potential for assessing the anaerobic ability of athletes, as well as the changes in this ability as a result of training.

Anaerobic fitness, during growth and development of athletes, has not received the same attention from researchers as aerobic fitness. This is surprising given the level of anaerobic energy used daily during childhood and adolescence (Sargeant, 1989). During physical activity and sport, the child is spontaneously more attracted to short-burst movements than to long-term activities. Many authors suggest that the pediatric population spends most of its time in play and recreation activities that require short bursts of activity followed by rest. It relies more heavily on a supply of energy from anaerobic means than aerobic means than aerobic means (Van Praagh, 2000; 2007; Van Praagh, Dole, 2002). Short-term high intensity tests are good tests for assessment of the functional capacity in children because they are similar to their habitual physical activity patterns in duration and intensity (Sargeant,1989). According to Amstrong et al, 2001, a small number of longitudinal studies examine the anaerobic performance of children using the Wingate Anaerobic Test.

The results of current study of young soccer players show that peak power and average power are similar to those found in elite soccer players (Al-Hazzaa et al, 2001; Gharbi Z et al, 2005; Smith, Hill,1991) or slightly lower (Ponorac et al, 2007; Popadic,2009). This contradicts the opinion of Van Praagh (2007) about the lesser ability of children to generate mechanical energy from chemical energy sources during short-term high-intensity work or exercise. The training protocol applied in our study had positive effects on athletes' performance, with peak power and average power results showing relatively close improvements, while the fatigue index (PD \%) remained unchanged.

The opinions of the experts about which of the parameters considered in the WAnT are the best for the assessment of the anaerobic ability of athletes are not uniform. According to Driss, Vandewalle (2013) the fatigue index is the least reliable of the three Wingate test indices, and its validity was questioned as it largely depends on the aerobic performance. Consequently, peak power and mean power output were the main topics considered and discussed in most of the studies (Vandevalle et al,1985; Armstrong et al, 2001; Peev,2017). Nonetheless, the validity of mean power as an index of anaerobic capacity is as questionable as the validity of the fatigue index. Therefore, peak power during Wingate test is probably the only index that merits to be measured (Driss, Vandewalle, 2013). This supports our view that expanding the physiological parameters measured and evaluated during the WAnT, would be necessary and useful for further implementations in laboratory protocols and sports practice.

The differences in calculated physiological parameters during the performance of the WAnT before (Test I) and after (Test II) following at the end of five months training period are more pronounced compared to the ergometric parameters, especially with regard to their contribution and participation in the anaerobic energy processes. It should be noted that energy for exercise is not merely the result of a series of energy systems switching on but rather the smooth blending and overlap of the body's three energy systems (alactic, lactic and aerobic). Therefore, we use the terms "anaerobic" and "aerobic" to mainly indicate the predominance of one or the other type of metabolic process.

The Wingate test allows power output and 
oxygen uptake to be investigated during various segments of the duration of the test.

Our measurements showed that at the beginning of the test, in the very first second, oxygen consumption is already available. Our results also indicate that as the pedalling time progresses $\mathrm{VO}_{2}$ rises, reaching the highest values 3-5 seconds before the completion of the test. At the end of the test, oxygen consumption reaches an average $86 \%$ of $\mathrm{VO}_{2}$ max. It has been estimated that even less than $1 \mathrm{sec}$ ond may be required to reach peak ATP turnover from ATP-PC stores (Smith, Hill 1991). It has been accepted that ATP-PC contribution is $100 \%$ until peak power was achieved. Nordhof et al, (2010), Serresse et al (1988) considered that these metabolic changes are happening at the 2,5 s point during the test. Sargeant and Dolan (1987) reported that the peak power occured 1-4 s into the exercise. Our data about the time to reach PP obtained from the Wingate test software is shorter $(2,082 \pm 0,868 \mathrm{~s})$ and is unaffected by the training done during the period of 5 months.

The method that we offer can only provide important guidance on the relationship between the metabolic processes related to the involvement sequence of energy systems when athletes are performing the Wingate test. It has been already noted that our goal was to look for the possible specific interactions in these relationships, which in turn reflect the changes in the anaerobic ability of the athletes in response to the training.

The conclusions for the amount of anaerobic energy supply during the performance of the WAnT could be made on the magnitude of the oxygen deficit, based on the differences in the mean values obtained before and after 5 months of training.

At the beginning of the Wingate test the energy for the initial exercise load is mainly provided by the anaerobic mechanisms and gradually the aerobic participation takes on an increasing share. The relationship between anaerobic and aerobic estimates of the participation of the energy systems shows a striking difference when comparing the data obtained before and after 5 months of training period. In our study the test results in response to the applied speed/power and endurance training protocol indicated a substantial improvement in the anaerobic abilities of the soccer players as follows: the oxygen deficit was higher by $32.4 \%(\alpha<0.001)$ and the participants were pedaling four seconds longer under the conditions of $\mathrm{O}_{2}$ deficit. An increase in the anaerobic capacity under the influence of anaerobic training was observed also by Medbo (1991). Medbo and Burgers (1990) reported a 10\% increase in accumulated oxygen deficit after six weeks of anaerobic training in a group of five male and seven female athletes.

The payoff of the $\mathrm{O}_{2}$ deficit accumulated during the first part of the test was done by the $\mathrm{O}_{2}$ excess overload measured during the Wingate exercise. During the Wingate test performed before the training the payoff starts from the 16th second forwards and after the training it starts four seconds later. Also, a significantly higher $\mathrm{O}_{2}$ excess volume, by $9.1 \%$, was established during the Wingate test after the training. The plausible explanation of this finding might be that less energy consumption is required for the restoration of homeostasis and this potentially could improve the options for a subsequent exercise. In the current study the smaller percentage increase in the $\mathrm{VO}_{2}$ uptake by $6,9 \%$ compared to the increase in the total power output by $16,4 \%$ could support the assumption that a greater metabolic efficiency is one of the plausible outcomes of the training.

The positive changes in the activation of the energy systems during the 30 -second supramaximal test could markedly alter the percentage of the participation of each system, as it was increased in favor of $\mathrm{O}_{2}$ deficiency by $80.3 \%(\alpha<0.001)$. According to our calculations the watts in $\%$ performed by the young 
soccer players on the basis of the anaerobic energy supply augmented from $37,1 \pm 11,8 \%$ of the total power output during the first Wingate test examination to $42,2 \pm 10,5 \%$ of the total power output after the 5-month training. Therefore, in this study the magnitude of the aerobic contribution to the 30 -s high power Wingate exercise task has been estimated to be near $60 \%$.

Most of the researchers in the field of accumulated oxygen debt (AOD) reported significantly lower magnitude of the aerobic contribution in the Wingate test by 40\% (Medbø, Tabata, 1985), by 28\% (Boulai et al, 1985), by $20 \%$ (Beneke et al, 2002), by $16 \%$ (Smith, Hill, 1991). According to Granier, Mercier (1995) the participation of aerobic processes is very significant and depends on the specificity of the particular sports investigated and might vary from $28 \%$ in sprinters to $45 \%$ in endurance sports. Gastin, 2001 provided the explanation that the possibility of a greater contribution from aerobic energy sources might occur in individuals who have a high $\mathrm{VO}_{2}$ max, faster $\mathrm{O}_{2}$ uptake kinetics, and low anaerobic powers. These characteristics are most likely to be found in well-trained endurance athletes.

Meldrum (2004) reported an anaerobic contribution of $28 \%$ to supramaximal exercise bout in groups of pubertal boys. Inbar O, BarOr O (1986) suggested that the biochemical correlates of such low anaerobic performance in children could be related to their lower maximal lactate concentration in muscle and blood, lower rate of anaerobic glycolysis and lower levels of acidosis during maximal exercise. It is noteworthy that the mechanisms of relatively deficient anaerobic characteristics in children are not quite clear.

The limitations of the present study are the small sample size, the limited number of young soccer teams in the country and the difficulty in obtaining informed consent forms from the parents.

\section{CONCLUSIONS}

The proposed method is focussed on investigating the relationship between the energy systems contributing to the performance of athletes during Wingate Test. The method is based on a certain number of easy to execute and obtain measurements, has clear practical value, but does not allow in-depth theoretical analysis. The physiological indicators can be defined on the basis of simultaneous registration the ergometric and oxygen uptake data of the Wingate test, allowing precise assessment of the relative participation of the energy systems when performing supramaximal exercise load. The results indicate that the physiological parameters are of high sensitivity and reflect strong associations with the metabolic changes in response to the training modalities. The method is easy to implement. It allows a quick analysis of the individual results and its application in exercise physiology laboratories and the sports practice could be beneficial.

\section{ACKNOWLEDGEMENTS}

The authors would like to express gratitude to P.Peev and members of staff of the high performance physiology laboratory at the Center for Scientific and Applied Research in Sport, National Sports Academy "Vassil Levski", Sofia, Bulgaria.

\section{REFERENCES}

Al-Hazzaa,H.M., Almuzaini,u.s., AlRefaee,S.A., Sulaiman, M.A., Dafterdar M.Y., Al-Ghamedi, A., Al-Khuraiji, K.N. (2001). Aerobic and anaerobic power characteristics of Saudi elite soccer player. J Sports Med Phys Fitness. Mar; 41(1):54-61

Armstrong, N., Welsman, J. R. \& Chia, M.Y.H.(2001). Short term power output in relation to growth and maturation. British $J$ Sports Med 35, 118-124.

Beneke, R., Pollmann, C., Bleif, I., Leithäuser, R.M., Hütler, M. (2002) How anaerobic is the Wingate Anaerobic Test for humans. Eur $J$ 
Appl Physiol 87: 388-392.

Bertuzzi, R.C., Franchini, E., Ugrinowitsch, C., Kokubun, E., Lima-Silva, A.E., Pires, .FO., et al. (2010). Predicting MAOD using only a supramaximal exhaustive test. Int J Sports Med; 31: 477-481.

Boulay, M.R., Lotrie, G., Simonau, J.A., Hamel, P., Leblanc, C., Bouchard, C. (1985). Specificity of anaerobic and aerobic work capacity and power. Int J Sports Med 6:325-328.

Driss, T., Vandewalle, H. (2013). The measurement of maximal (anaerobic) power output on a cycle ergometer: A critical review, Biomed research internationa, Article ID 589361, 40.

Gastin, P.B. (2001). Energy System Interaction and Relative Contribution During Maximal Exercise Sports Med; 31 (10): 725-741.

Gharbi, Z., Dardouri,W., Haj-Sassi, R., Chamari, K., Souissi, N. (2015). Aerobic and anaerobic determinants of repeated sprint ability in team sports athletes. Biol. Sport 32:207212.

Granier, P., Mercier, J.(1995). Aerobic and anaerobic contribution to Wingate test performance in sprint and middle distance runners. Eur J. Appl.Physiol., 70(1),58-65.

Green, S. (1995). Maximal oxygen deficit of sprint and middle distance runners. Eur

J Appl Physiol 70: 192-193.

Inbar, O., Bar-Or, O., Skinner, J.S.(1996). The Wingate anaerobic test. Champaign: $\mathrm{Hu}$ man Kinetics Books.

Inbar, O., Bar-Or, O. (1986). Anaerobic characteristics in male children and adolescents. Medicine and Science in Sports and Exercise. 18(3):264-9.

Medbo, J.I., Mohn, A.C., Tabata, I., Bahr, R.,Vaage, O.,Sejersted, O.M. (1988). Anaerobic capacity determined by maximal accumulated O2 deficit. J Appl Physiol.;64(1):50-60.

Medbo, J.I, Tabata, I. (1989). Relative importance of aerobic and anaerobic energy release during short-lasting exhausting bicycle exercise. J Appl Physiol.;67(5):1881-1886.
Medbo, J.I., Tabata, I. (1993). Anaerobic energy release in working muscle during $30 \mathrm{~s}$ to 3 min of exhausting bicycling. J Appl Physiol.;75(4):1654-1660.

Medbo J.I.,Burgers,S. (1990). Effect of training on the anaerobic capacity. Med Sci Sports Exerc. Aug; 22(4):501-7.

Medbo, J.I.,Tabata,I. (1985) Relative importance of aerobic and anaerobic energy release during short-lasting exhausting bicycle exrcise. J Appl Physiol, 67(5):1881-6

Meldrum, K. J. (2004). Measuring anaerobic performance in children using accumulated oxygen deficit. Dissertation submitted for the degree of Doctor of Philosophy, School of Human Movement, Recreation and Performance Victoria University.

Noordhof, D.A., de Koning, J.J., Foster, C. (2010). The maximal accumulated oxygen deficit method: a valid and reliable measure of anaerobic capacity? Sports Med.;40(4):285302.

Noordhof, D.A., Skiba, Ph., de Koning, J.J. (2013). Determining Anaerobic Capacity in Sporting Activities. Int J Sports Physiol Performance, 8,475-482.

Peev, P. (2017). Izsledvane na metodicheski podhodi pd vuzdeistvie vurhu razvitieto na skorostnata izdrujlivost pri 13-14 godishni futbolisti. Disertation PhD, NSA, Sofia.

Ponorac.,N., Matavulj, A., Rajkovaca, Z., Kavacevic., P. (2007). The assessment of anerobic capacity in athletes of various sports. Medinnski pregled 60: 427 -430.

Popadic Gacesa, J.Z

Barak, O.F., Grujic, N.G .(2009). Maximal anaerobic power test in athletes of different sport disciplines. $J$ Strength Cond Res. May;23(3):751-5.

Sargeant, A.J. (1989). Short term muscle power in children and adolescents. In Advances in Pediatric Sport Science, ed. Bar-Or, O. pp. 41 - 64. Human Kinetics, Champaign, Illinois. 
Sargeant, A.J., Dolan, P.(1987). Effect of prior exercise on maximal short-term power output in humans. J Appl Physiol; 63: 147580.

Serresse, O., Lortie, G., Bouchard, C., Boulay, M.R. (1988). Estimation of the contribution of the various energy systems during maximal work of short duration. Int $J$ Sports Med.;9:456-460.

Smith, J.C., Hil, D.W. (1991) Contribution of energy systems during a Wingate power test.

Br J Sports Med 25:196-199.

Van Praagh, E. (2000). Development of anaerobic fiinction during childhood and adolescence. Pediatric Exercise Science 12, 150-173.Van Praagh, E., DOLE. E. (2002).
Short-Term Muscle Power During Growth and Maturation. Sports Medicine 32, 701-728.

Van Praagh, E . (2007). Anaerobic fitness tests: what are we measuring?Med Sport Sci; 50:26-45.

Vandewalle, H., Peres, G., Heller, J., Monod, H. (1985). All out anaerobic capacity tests on cycle ergometers. A comparative study on men and women. Eur J Appl Physiol and Occup Physiol, 54, 222-229.

Zagatto, A. M., Bertuzzi, R., Miyagi,W.E., Padulo,J.,Papoti,M.(2016) MAOD determination in a single supramaximal test: a study on the reliability and effects of supramaximal intensities. Int J Sports Med.Aug; 37(9):700-7.

\section{Correspondence:}

Dorothea Stefanova

National Sports Academy "Vassil Levski" Studentski grad, 21, Acad. Stefan Mladenov Str.

Sofia 1700, Bulgaria E-mail: dorstef@mail.bg 